\title{
ON REDUCIBLE MONODROMY REPRESENTATIONS OF SOME GENERALIZED LAMÉ EQUATION
}

\author{
ZHIJIE CHEN, TING-JUNG KUO, CHANG-SHOU LIN, AND KOUICHI TAKEMURA
}

\begin{abstract}
In this note, we compute the explicit formula of the monodromy data for a generalized Lamé equation when its monodromy is reducible but not completely reducible. We also solve the corresponding Riemman-Hilbert problem.
\end{abstract}

\section{INTRODUCTION}

Throughout the paper, we use the notations $\omega_{0}=0, \omega_{1}=1, \omega_{2}=\tau$, $\omega_{3}=1+\tau$ and $\Lambda_{\tau}=\mathbb{Z}+\mathbb{Z} \tau$, where $\tau \in \mathbb{H}=\{\tau \mid \operatorname{Im} \tau>0\}$. Define $E_{\tau}:=\mathbb{C} / \Lambda_{\tau}$ to be a flat torus in the plane and $E_{\tau}[2]:=\left\{\frac{\omega_{k}}{2} \mid 0 \leq k \leq 3\right\}+\Lambda_{\tau}$ to be the set consisting of the lattice points and 2-torsion points in $E_{\tau}$.

Let $\wp(z)=\wp(z \mid \tau)$ be the Weierstrass elliptic function with periods $\Lambda_{\tau}$, defined by

$$
\wp(z \mid \tau):=\frac{1}{z^{2}}+\sum_{\omega \in \Lambda_{\tau} \backslash\{0\}}\left(\frac{1}{(z-\omega)^{2}}-\frac{1}{\omega^{2}}\right),
$$

and $e_{k}=e_{k}(\tau):=\wp\left(\frac{\omega_{k}}{2} \mid \tau\right), k \in\{1,2,3\} . \operatorname{Let} \zeta(z)=\zeta(z \mid \tau):=-\int^{z} \wp(\xi \mid \tau) d \xi$ be the Weierstrass zeta function, which is an odd meromorphic function with two quasi-periods:

$$
\eta_{1}(\tau)=\zeta(z+1 \mid \tau)-\zeta(z \mid \tau), \quad \eta_{2}(\tau)=\zeta(z+\tau \mid \tau)-\zeta(z \mid \tau)
$$

In this note, we study the following generalized Lamé equation (GLE):

$$
y^{\prime \prime}(z)=\left[\begin{array}{l}
\sum_{k=0}^{3} n_{k}\left(n_{k}+1\right) \wp\left(z+\frac{\omega_{k}}{2}\right)+\frac{3}{4}(\wp(z+p) \\
+\wp(z-p))+A(\zeta(z+p)-\zeta(z-p))+B
\end{array}\right] y(z) \text { in } E_{\tau},
$$

where $n_{k} \in \mathbb{N} \cup\{0\}$ for all $k, A, B \in \mathbb{C}$ and $\pm p \notin E_{\tau}[2]$ are always assumed to be apparent singularities (i.e. non-logarithmic). Under this assumption, $B$ is determined by $(p, A)$ as follows (see [2]):

$$
B=A^{2}-\zeta(2 p) A-\frac{3}{4} \wp(2 p)-\sum_{k=0}^{3} n_{k}\left(n_{k}+1\right) \wp\left(p+\frac{\omega_{k}}{2}\right) .
$$

We are interested in GLE (1.2), which was studied in [10, 2], because it has a deep relation with the well-known Panlevé VI equation. Indeed, if $(A(\tau), B(\tau), p(\tau))$ depends on $\tau$ suitably such that GLE (1.2) preserves the monodromy as $\tau$ deforms, then $p(\tau)$ satisfies the elliptic form of Panlevé VI equation. See [2] or Section 3. Note that by letting $x=\wp(z)$, GLE 
(1.2) can be projected to a new equation on $\mathbb{C P}^{1}$, which is a second order Fuchsian equation with five singular points $\left\{e_{1}, e_{2}, e_{3}, \wp(p), \infty\right\}$ with $\wp(p)$ being apparent. Such type of ODEs on $\mathbb{C P}^{1}$ have been widely studied in the literature. However, the monodromy of this new ODE are not easy to compute. Therefore, it is more convenient for us to study GLE (1.2) in $E_{\tau}$ directly as long as the monodromy is concerned.

The monodromy representation of GLE (1.2) is a homomorphism $\rho$ : $\pi_{1}\left(E_{\tau} \backslash\left(E_{\tau}[2] \cup\left(\{ \pm p\}+\Lambda_{\tau}\right)\right), q_{0}\right) \rightarrow S L(2, \mathbb{C})$, where $q_{0} \notin E_{\tau}[2] \cup(\{ \pm p\}+$ $\left.\Lambda_{\tau}\right)$ is a base point. Let $\gamma_{ \pm} \in \pi_{1}\left(E_{\tau} \backslash\left(E_{\tau}[2] \cup\left(\{ \pm p\}+\Lambda_{\tau}\right)\right), q_{0}\right)$ be a simple loop encircling $\pm p$ counterclockwise respectively, and $\ell_{j}, j=1,2$, be two fundamental cycles of $E_{\tau}$ connecting $q_{0}$ with $q_{0}+\omega_{j}$ such that $\ell_{j}$ does not intersect with $L+\Lambda_{\tau}$ (here $L$ is the straight segment connecting $\pm p$ ) and satisfies

$$
\gamma_{+} \gamma_{-}=\ell_{1} \ell_{2} \ell_{1}^{-1} \ell_{2}^{-1} \text { in } \pi_{1}\left(E_{\tau} \backslash\left(\{ \pm p\}+\Lambda_{\tau}\right), q_{0}\right) .
$$

Since the local exponents of (1.2) at $\pm p$ are $-\frac{1}{2}$ and $\frac{3}{2}$ and $\pm p \notin E_{\tau}$ [2] are apparent singularities, we always have

$$
\rho\left(\gamma_{ \pm}\right)=-I_{2}
$$

For any $k \in\{0,1,2,3\}$, the local exponents of GLE (1.2) at $\omega_{k} / 2$ are $-n_{k}$ and $n_{k}+1$ with $n_{k} \in \mathbb{Z}$. Since the potential of GLE (1.2) is even elliptic, the local monodromy matrix of GLE (1.2) at $\omega_{k} / 2$ is $I_{2}$ (see e.g. [10, Lemma 2.2]). Therefore, the monodromy group of GLE (1.2) is generated by $\left\{-I_{2}, \rho\left(\ell_{1}\right), \rho\left(\ell_{2}\right)\right\}$. Together with (1.4) and (1.5), we immediately obtain $\rho\left(\ell_{1}\right) \rho\left(\ell_{2}\right)=\rho\left(\ell_{2}\right) \rho\left(\ell_{1}\right)$, which implies that the monodromy group of GLE (1.2) is always abelian and hence reducible, i.e. all the monodromy matrices have at least a common eigenfunction. Clearly there are two cases:

(a) Completely reducible, i.e. all the monodromy matrices have two linearly independent common eigenfunctions. This case has been well studied in [4].

(b) Not completely reducible, i.e. the space of common eigenfunctions is of dimension 1: Up to a common conjugation,

$$
\rho\left(\ell_{1}\right)=\varepsilon_{1}\left(\begin{array}{ll}
1 & 0 \\
1 & 1
\end{array}\right), \quad \rho\left(\ell_{2}\right)=\varepsilon_{2}\left(\begin{array}{ll}
1 & 0 \\
C & 1
\end{array}\right),
$$

where $\varepsilon_{1}, \varepsilon_{2} \in\{ \pm 1\}$ and $C \in \mathbb{C} \cup\{\infty\}$. Remark that if $C=\infty$, then (1.6) should be understood as

$$
\rho\left(\ell_{1}\right)=\varepsilon_{1}\left(\begin{array}{ll}
1 & 0 \\
0 & 1
\end{array}\right), \quad \rho\left(\ell_{2}\right)=\varepsilon_{2}\left(\begin{array}{ll}
1 & 0 \\
1 & 1
\end{array}\right) .
$$

In this case, $C$ is called the monodromy data of GLE (1.2).

Remark that for the projective ODE of GLE (1.2) on $\mathbb{C P}^{1}$, its monodromy representation is irreducible if and only if Case (a) occurs, and reducible if and only if Case (b) occurs. Most of the references in the literature are 
devoted to the case of irreducible representation on $\mathbb{C P}^{1}$, but very few are devoted to studying reducible representation.

We are interested in the explicit formula of the monodromy data $C$ when Case (b) occurs. This problem is important but challenging for general $n_{k}$, which will be studied in a future paper. In this note, we focus on the special case $\left(n_{0}, n_{1}, n_{2}, n_{3}\right)=(1,0,0,0)$, i.e. GLE

$$
y^{\prime \prime}(z)=\left[\begin{array}{c}
2 \wp(z)+\frac{3}{4}(\wp(z+p)+\wp(z-p)) \\
+A(\zeta(z+p)-\zeta(z-p))+B
\end{array}\right] y(z) \text { in } E_{\tau} .
$$

Our first result is following.

Theorem 1.1. Fix $\tau \in \mathbb{H}$ and $p \notin E_{\tau}[2]$. If the monodromy of GLE (1.7) is not completely reducible, then the monodromy data $C$ satisfies either

$$
\wp(p \mid \tau)=\frac{2 g_{3}(C-\tau)^{3}-4\left(C \eta_{1}-\eta_{2}\right)^{3}-g_{2}\left(C \eta_{1}-\eta_{2}\right)(C-\tau)^{2}}{(C-\tau)\left[12\left(C \eta_{1}-\eta_{2}\right)^{2}-g_{2}(C-\tau)^{2}\right]},
$$

with $\left(\varepsilon_{1}, \varepsilon_{2}\right)=(1,1)$ or

$$
\wp(p \mid \tau)=\frac{\left(\frac{g_{2}}{2}-3 e_{k}^{2}\right)\left(C \eta_{1}-\eta_{2}\right)+\frac{g_{2}}{4} e_{k}(C-\tau)}{3 e_{k}\left(C \eta_{1}-\eta_{2}\right)+\left(\frac{g_{2}}{2}-3 e_{k}^{2}\right)(C-\tau)},
$$

for some $k \in\{1,2,3\}$ with

$$
\left(\varepsilon_{1}, \varepsilon_{2}\right)=\left\{\begin{array}{l}
(1,-1) \text { if } k=1 \\
(-1,1) \text { if } k=2 \\
(-1,-1) \text { if } k=3
\end{array}\right.
$$

Here $g_{2}=g_{2}(\tau)$ and $g_{3}=g_{3}(\tau)$ are the coefficients of

$$
\wp^{\prime}(z \mid \tau)^{2}=4 \wp(z \mid \tau)^{3}-g_{2}(\tau) \wp(z \mid \tau)-g_{3}(\tau)=4 \prod_{k=1}^{3}\left(\wp(z \mid \tau)-e_{k}(\tau)\right) .
$$

The formulas (1.8)-(1.9) first appeared in [10, (3.68)-(3.69)] without detailed proofs and later was obtained in [3] independently, as explicit expressions of Riccati type solutions of Painlevé VI equation. But their connection with the monodromy data seems not be well addressed. Theorem 1.1 can be proved directly without applying Painlevé VI equation; see Section 2.

Conversely, it is natural to consider the following Riemann-Hilbert problem: For fixed $\tau \in \mathbb{H}$ and $p \notin E_{\tau}[2]$, and given any $C$ satisfying (1.8) or (1.9), whether there exists GLE (1.7) (i.e. exist $A, B \in \mathbb{C}$ ) such that this $C$ is its monodromy data? This problem is fundamental but seems not be settled. Our second main result is to answer this question positively.

Theorem 1.2. Fix $\tau \in \mathbb{H}$ and $p \notin E_{\tau}[2]$.

(1) If $C \in \mathbb{C} \cup\{\infty\}$ satisfies the cubic equation (1.8), then there exists $A \in \mathbb{C}$ (and $B$ is given by $(p, A)$ via (1.3) with $\left(n_{0}, n_{1}, n_{2}, n_{3}\right)=$ 
$(1,0,0,0))$ such that for the correpsonding GLE (1.7), up to a common conjugation,

$$
\rho\left(\ell_{1}\right)=\left(\begin{array}{ll}
1 & 0 \\
1 & 1
\end{array}\right), \quad \rho\left(\ell_{2}\right)=\left(\begin{array}{ll}
1 & 0 \\
C & 1
\end{array}\right) .
$$

(2) Fix $k \in\{1,2,3\}$. If $C \in \mathbb{C} \cup\{\infty\}$ satisfies the equation (1.9), then there exists $A \in \mathbb{C}$ such that for the correpsonding GLE (1.7), up to a common conjugation,

$$
\rho\left(\ell_{1}\right)=\varepsilon_{1}\left(\begin{array}{ll}
1 & 0 \\
1 & 1
\end{array}\right), \quad \rho\left(\ell_{2}\right)=\varepsilon_{2}\left(\begin{array}{ll}
1 & 0 \\
C & 1
\end{array}\right),
$$

where $\left(\varepsilon_{1}, \varepsilon_{2}\right)$ is given by (1.10).

If we know that the number of $A^{\prime}$ 's with the monodromy of the corresponding GLE (1.7) being not completely reducible is 6, then Theorem 1.2 might follow from Theorem 1.1. However, this assumption can not hold for all $(p, \tau)$, and the proof of Theorem 1.2 becomes subtle. In Section 3, we will present a proof by applying the connection between GLE (1.7) and Painlevé VI equation. Theorem 1.1 will be proved in Section 2.

\section{Proof of Theorem 1.1}

The purpose of this section is to prove Theorem 1.1. Let $y_{1}(z)$ be a common eigenfunction. Then it is known (cf. [4]) that $y_{1}(z)$ can be expressed as

$$
y_{1}(z)=e^{\left(r \eta_{1}+s \eta_{2}\right) z} \frac{\sigma\left(z-a_{1}\right) \sigma\left(z-a_{2}\right)}{\sigma(z)^{2}} \frac{\sigma(z)}{[\sigma(z-p) \sigma(z+p)]^{\frac{1}{2}}},
$$

where $(r, s)$ is determined by

$$
r+s \tau=a_{1}+a_{2}, r \eta_{1}+s \eta_{2}=\zeta\left(a_{1}\right)+\zeta\left(a_{2}\right),
$$

and $a_{1}, a_{2} \notin \Lambda_{\tau}$ satisfy

$$
\begin{aligned}
& {\left[\zeta\left(a_{i}+p\right)+\zeta\left(a_{i}-p\right)-2 \zeta\left(a_{i}\right)\right] } \\
= & 2\left[\zeta\left(a_{i}-a_{j}\right)+\zeta\left(a_{j}\right)-\zeta\left(a_{i}\right)\right],\{i, j\}=\{1,2\} .
\end{aligned}
$$

Here $\sigma(z)=\sigma(z \mid \tau)$ is the Weierstrass sigma function defined by $\frac{\sigma^{\prime}(z)}{\sigma(z)}:=$ $\zeta(z)$. It is known that $\sigma(z)$ is an odd entire function with simple zeros only at the lattice points $\Lambda_{\tau}$. Remark that (2.3) can be easily obtained by inserting (2.1) into GLE (1.7).

Proof of Theorem 1.1. Suppose the monodromy representation of GLE (1.7) is not completely reducible, i.e. the space of common eigenfunctions is of dimension 1. Then $y_{1}(-z)= \pm y_{1}(z)$ (because $y_{1}(-z)$ is also a common eigenfunction), i.e.

$$
\left\{a_{1}, a_{2}\right\}=\left\{-a_{1},-a_{2}\right\} \text { in } E_{\tau} .
$$


Remark that $\frac{\sigma(z)^{2}}{\sigma(z-p) \sigma(z+p)}$ is even elliptic. Since $\ell_{j}$ does not intersect with $L+\Lambda_{\tau}$, the function $\frac{\sigma(z)}{[\sigma(z-p) \sigma(z+p)]^{\frac{1}{2}}}$ is invariant under analytic continuation along $\ell_{j}$ (see [4]), i.e.

$$
\ell_{j}^{*} \frac{\sigma(z)}{[\sigma(z-p) \sigma(z+p)]^{\frac{1}{2}}}=\frac{\sigma(z)}{[\sigma(z-p) \sigma(z+p)]^{\frac{1}{2}}}, j=1,2 .
$$

Here we denote by $\ell_{j}^{*} f(z)$ to be the function obtained from $f(z)$ under analytic continuation along $\ell_{j}$. By (2.4) there are two cases.

Case 1. $a_{j} \notin E_{\tau}[2]$ for $j=1,2$, then $a_{2}=-a_{1}$.

Then $(r, s)=(0,0)$, i.e.

$$
y_{1}(z)=\frac{\sigma\left(z-a_{1}\right) \sigma\left(z+a_{1}\right)}{\sigma(z)^{2}} \frac{\sigma(z)}{[\sigma(z-p) \sigma(z+p)]^{\frac{1}{2}}} .
$$

By (2.5) and the transformation law

$$
\sigma\left(z+\omega_{j}\right)=-e^{\left(z+\omega_{j} / 2\right) \eta_{j}} \sigma(z), \quad j=1,2,
$$

it is easy to prove that

$$
\ell_{j}^{*} y_{1}(z)=y_{1}(z) \text { for } j=1,2 .
$$

Therefore, $y_{1}(z)^{2}$ is even elliptic, i.e. up to a constant,

$$
y_{1}(z)^{-2}=\frac{\wp(z)-\wp(p)}{\left(\wp(z)-\wp\left(a_{1}\right)\right)^{2}}
$$

On the other hand, it follows from the addition formulas

and (2.3) that

$$
\begin{gathered}
\zeta(u+v)+\zeta(u-v)-2 \zeta(u)=\frac{\wp^{\prime}(u)}{\wp(u)-\wp(v)}, \\
\zeta(2 u)-2 \zeta(u)=\frac{\wp^{\prime \prime}(u)}{2 \wp^{\prime}(u)}
\end{gathered}
$$

$$
\frac{\wp^{\prime}\left(a_{1}\right)}{\wp\left(a_{1}\right)-\wp(p)}=2\left[\zeta\left(2 a_{1}\right)-2 \zeta\left(a_{i}\right)\right]=\frac{\wp^{\prime \prime}\left(a_{1}\right)}{\wp^{\prime}\left(a_{1}\right)},
$$

i.e.

$$
\wp(p)=\wp\left(a_{1}\right)-\frac{\wp^{\prime}\left(a_{1}\right)^{2}}{\wp^{\prime \prime}\left(a_{1}\right)} .
$$

From here and (2.8), it is easy to see that the residues of $y_{1}(z)^{-2}$ at $\pm a_{1}$ are both 0 , so

$$
\begin{gathered}
y_{1}(z)^{-2}=c_{3}\left[\wp\left(z-a_{1}\right)+\wp\left(z+a_{1}\right)-2 \wp\left(a_{1}\right)\right] \\
\text { where } c_{3}=\frac{\wp\left(a_{1}\right)-\wp(p)}{\wp^{\prime}\left(a_{1}\right)^{2}} \text {. Then } \\
\chi \chi(z):=\int_{0}^{z} \frac{1}{y_{1}(\xi)^{2}} d \xi=-c_{3}\left(\zeta\left(z-a_{1}\right)+\zeta\left(z+a_{1}\right)+2 \wp\left(a_{1}\right) z\right),
\end{gathered}
$$


and so $\chi(z)$ is quasi-periodic with two quasi-periods:

$$
\begin{gathered}
\chi_{1}=\chi(z+1)-\chi(z)=-2 c_{3}\left(\eta_{1}+\wp\left(a_{1}\right)\right), \\
\chi_{2}=\chi(z+\tau)-\chi(z)=-2 c_{3}\left(\eta_{2}+\wp\left(a_{1}\right) \tau\right) .
\end{gathered}
$$

Define

$$
C:=\frac{\chi_{2}}{\chi_{1}}=\frac{\eta_{2}+\wp\left(a_{1}\right) \tau}{\eta_{1}+\wp\left(a_{1}\right)}
$$

and $y_{2}(z):=y_{1}(z) \chi(z)$. Since $y_{1}(z)$ is a solution of GLE (1.7) and $\chi(z)=$ $\int_{0}^{z} \frac{1}{y_{1}(\xi)^{2}} d \xi$, it is easy to see that $y_{2}(z)$ is a linearly independent solution of GLE (1.7) with respect to $y_{1}(z)$. Recalling (2.7) and that $y_{2}(z)$ is not a common eigenfunction, we know that $\chi_{1}, \chi_{2}$ can not vanish simultaneously. Consequently, if $\chi_{1}=0$, then $\chi_{2} \neq 0, C=\infty$ and so

$$
\begin{gathered}
\ell_{1}^{*}\left(\begin{array}{c}
\chi_{2} y_{1}(z) \\
y_{2}(z)
\end{array}\right)=\left(\begin{array}{c}
\chi_{2} y_{1}(z) \\
y_{2}(z)
\end{array}\right), \text { i.e. } \rho\left(\ell_{1}\right)=I_{2}, \\
\ell_{2}^{*}\left(\begin{array}{c}
\chi_{2} y_{1}(z) \\
y_{2}(z)
\end{array}\right)=\left(\begin{array}{ll}
1 & 0 \\
1 & 1
\end{array}\right)\left(\begin{array}{c}
\chi_{2} y_{1}(z) \\
y_{2}(z)
\end{array}\right) \text {, i.e. } \rho\left(\ell_{2}\right)=\left(\begin{array}{ll}
1 & 0 \\
1 & 1
\end{array}\right) .
\end{gathered}
$$

If $\chi_{1} \neq 0$, then $C \neq \infty$ and so

$$
\begin{aligned}
& \ell_{1}^{*}\left(\begin{array}{c}
\chi_{1} y_{1}(z) \\
y_{2}(z)
\end{array}\right)=\left(\begin{array}{ll}
1 & 0 \\
1 & 1
\end{array}\right)\left(\begin{array}{c}
\chi_{1} y_{1}(z) \\
y_{2}(z)
\end{array}\right), \text { i.e. } \rho\left(\ell_{1}\right)=\left(\begin{array}{ll}
1 & 0 \\
1 & 1
\end{array}\right), \\
& \ell_{2}^{*}\left(\begin{array}{c}
\chi_{1} y_{1}(z) \\
y_{2}(z)
\end{array}\right)=\left(\begin{array}{ll}
1 & 0 \\
C & 1
\end{array}\right)\left(\begin{array}{c}
\chi_{1} y_{1}(z) \\
y_{2}(z)
\end{array}\right), \text { i.e. } \rho\left(\ell_{2}\right)=\left(\begin{array}{ll}
1 & 0 \\
C & 1
\end{array}\right) .
\end{aligned}
$$

Clearly (2.10) gives

$$
\wp\left(a_{1}\right)=\frac{C \eta_{1}-\eta_{2}}{\tau-C} .
$$

Inserting this into (2.9) and using $\wp^{\prime}\left(a_{1}\right)^{2}=4 \wp\left(a_{1}\right)^{3}-g_{2} \wp\left(a_{1}\right)-g_{3}, \wp^{\prime \prime}\left(a_{1}\right)=$ $6 \wp\left(a_{1}\right)^{2}-g_{2} / 2$, we easily obtain

$$
\wp(p)=\frac{2 g_{3}(C-\tau)^{3}-4\left(C \eta_{1}-\eta_{2}\right)^{3}-g_{2}\left(C \eta_{1}-\eta_{2}\right)(C-\tau)^{2}}{(C-\tau)\left[12\left(C \eta_{1}-\eta_{2}\right)^{2}-g_{2}(C-\tau)^{2}\right]} .
$$

This proves 1.8$)$ with $\left(\varepsilon_{1}, \varepsilon_{2}\right)=(1,1)$.

Case 2. $a_{l} \in E_{\tau}[2]$ for some $l \in\{1,2\}$, then $\left\{a_{1}, a_{2}\right\}=\left\{\frac{\omega_{i}}{2}, \frac{\omega_{j}}{2}\right\}$ for some $i \neq j \in\{1,2,3\}$. Denote $\eta_{3}=\eta_{1}+\eta_{2}$.

Note from (2.2) that $r \eta_{1}+s \eta_{2}=\frac{1}{2}\left(\eta_{i}+\eta_{j}\right)$. Let $\{k\}=\{1,2,3\} /\{i, j\}$. By (2.5) and (2.6), it is easy to prove that

$$
\ell_{1}^{*} y_{1}(z)=(-1)^{k+m(k)} y_{1}(z), \quad \ell_{2}^{*} y_{1}(z)=(-1)^{k} y_{1}(z)
$$

Then $y_{1}(z)^{2}$ is even elliptic, i.e. up to a constant,

$$
y_{1}(z)^{-2}=\frac{\wp(z)-\wp(p)}{\left(\wp(z)-e_{i}\right)\left(\wp(z)-e_{j}\right)} .
$$


Again a direct computation shows that the residue of $y_{1}(z)^{-2}$ at $\frac{\omega_{i}}{2}, \frac{\omega_{j}}{2}$ are both 0 , so

$$
y_{1}(z)^{-2}=c_{3}\left(\wp\left(z-\frac{\omega_{i}}{2}\right)-e_{i}\right)+c_{4}\left(\wp\left(z-\frac{\omega_{j}}{2}\right)-e_{j}\right),
$$

where

$$
c_{3}=\frac{2\left(e_{i}-\wp(p)\right)}{\left(e_{i}-e_{j}\right) \wp^{\prime \prime}\left(\frac{\omega_{i}}{2}\right)}, \quad c_{4}=\frac{2\left(e_{j}-\wp(p)\right)}{\left(e_{j}-e_{i}\right) \wp^{\prime \prime}\left(\frac{\omega_{j}}{2}\right)} .
$$

By $\wp^{\prime \prime}\left(\frac{\omega_{i}}{2}\right)=6 e_{i}^{2}-g_{2} / 2, g_{2}=4\left(e_{k}^{2}-e_{i} e_{j}\right)$ and $e_{i}+e_{j}+e_{k}=0$, a direct computation gives

$$
\begin{aligned}
c_{3}+c_{4} & =\frac{2 g_{2}-12 e_{k}^{2}-12 e_{k} \wp(p)}{\wp^{\prime \prime}\left(\frac{\omega_{i}}{2}\right) \wp^{\prime \prime}\left(\frac{\omega_{j}}{2}\right)}, \\
c_{3} e_{i}+c_{4} e_{j} & =\frac{g_{2} e_{k}+12 \wp(p) e_{k}^{2}-2 g_{2} \wp(p)}{\wp^{\prime \prime}\left(\frac{\omega_{i}}{2}\right) \wp^{\prime \prime}\left(\frac{\omega_{j}}{2}\right)} .
\end{aligned}
$$

Define

$$
\chi(z):=\int_{0}^{z} \frac{1}{y_{1}(\xi)^{2}} d \xi=-c_{3}\left(\zeta\left(z-\frac{\omega_{i}}{2}\right)+e_{i} z\right)-c_{4}\left(\zeta\left(z-\frac{\omega_{j}}{2}\right)+e_{j} z\right),
$$

then it follows from the Lengrede relation $\tau \eta_{1}-2 \pi i=\eta_{2}$ that

$$
\begin{aligned}
\chi_{1} & =\chi(z+1)-\chi(z)=-\eta_{1}\left(c_{3}+c_{4}\right)-\left(c_{3} e_{i}+c_{4} e_{j}\right), \\
\chi_{2} & =\chi(z+\tau)-\chi(z)=-\eta_{2}\left(c_{3}+c_{4}\right)-\left(c_{3} e_{i}+c_{4} e_{j}\right) \tau \\
& =\tau \chi_{1}+2 \pi i\left(c_{3}+c_{4}\right) .
\end{aligned}
$$

Again we define

$$
C:=\frac{\chi_{2}}{\chi_{1}}=\tau+\frac{2 \pi i\left(c_{3}+c_{4}\right)}{\chi_{1}},
$$

and $y_{2}(z):=y_{1}(z) \chi(z)$. Then as above, we have the following conclusions: If $\chi_{1}=0$, then $\chi_{2} \neq 0, C=\infty$ and so

$$
\begin{gathered}
\ell_{1}^{*}\left(\begin{array}{c}
\chi_{2} y_{1}(z) \\
y_{2}(z)
\end{array}\right)=(-1)^{k+m(k)}\left(\begin{array}{c}
\chi_{2} y_{1}(z) \\
y_{2}(z)
\end{array}\right), \\
\ell_{2}^{*}\left(\begin{array}{c}
\chi_{2} y_{1}(z) \\
y_{2}(z)
\end{array}\right)=(-1)^{k}\left(\begin{array}{ll}
1 & 0 \\
1 & 1
\end{array}\right)\left(\begin{array}{c}
\chi_{2} y_{1}(z) \\
y_{2}(z)
\end{array}\right), \\
\text { i.e. } \rho\left(\ell_{1}\right)=(-1)^{k+m(k)} I_{2} \text { and } \rho\left(\ell_{2}\right)=(-1)^{k}\left(\begin{array}{ll}
1 & 0 \\
1 & 1
\end{array}\right) .
\end{gathered}
$$

If $\chi_{1} \neq 0$, then $C \neq \infty$ and so

$$
\begin{gathered}
\ell_{1}^{*}\left(\begin{array}{c}
\chi_{1} y_{1}(z) \\
y_{2}(z)
\end{array}\right)=(-1)^{k+m(k)}\left(\begin{array}{ll}
1 & 0 \\
1 & 1
\end{array}\right)\left(\begin{array}{c}
\chi_{1} y_{1}(z) \\
y_{2}(z)
\end{array}\right), \\
\ell_{2}^{*}\left(\begin{array}{c}
\chi_{1} y_{1}(z) \\
y_{2}(z)
\end{array}\right)=(-1)^{k}\left(\begin{array}{ll}
1 & 0 \\
C & 1
\end{array}\right)\left(\begin{array}{c}
\chi_{1} y_{1}(z) \\
y_{2}(z)
\end{array}\right),
\end{gathered}
$$




$$
\text { i.e. } \rho\left(\ell_{1}\right)=(-1)^{k+m(k)}\left(\begin{array}{ll}
1 & 0 \\
1 & 1
\end{array}\right) \text { and } \rho\left(\ell_{2}\right)=(-1)^{k}\left(\begin{array}{ll}
1 & 0 \\
C & 1
\end{array}\right) \text {. }
$$

By (2.11)-(2.12) we have

$$
\frac{C \eta_{1}-\eta_{2}}{\tau-C}=-\eta_{1}-\frac{\chi_{1}}{c_{3}+c_{4}}=\frac{c_{3} e_{i}+c_{4} e_{j}}{c_{3}+c_{4}}=\frac{\left(\frac{g_{2}}{2}-3 e_{k}^{2}\right) \wp(p)-\frac{g_{2}}{4} e_{k}}{3 e_{k} \wp(p)+3 e_{k}^{2}-\frac{g_{2}}{2}},
$$

and so

$$
\wp(p)=\frac{\left(\frac{g_{2}}{2}-3 e_{k}^{2}\right)\left(C \eta_{1}-\eta_{2}\right)+\frac{g_{2}}{4} e_{k}(C-\tau)}{3 e_{k}\left(C \eta_{1}-\eta_{2}\right)+\left(\frac{g_{2}}{2}-3 e_{k}^{2}\right)(C-\tau)} .
$$

This proves (1.9) with $\left(\varepsilon_{1}, \varepsilon_{2}\right)$ given by (1.10).

\section{Relation With Painlevé VI eQuation}

This section is devoted to the proof of Theorem 1.2. First we recall the relation between GLE (1.2) and Panlevé VI equation. The well-known Painlevé VI equation with four parameters $(\alpha, \beta, \gamma, \delta)(\operatorname{PVI}(\alpha, \beta, \gamma, \delta))$ is written as

$$
\begin{aligned}
\frac{d^{2} \lambda}{d t^{2}}= & \frac{1}{2}\left(\frac{1}{\lambda}+\frac{1}{\lambda-1}+\frac{1}{\lambda-t}\right)\left(\frac{d \lambda}{d t}\right)^{2}-\left(\frac{1}{t}+\frac{1}{t-1}+\frac{1}{\lambda-t}\right) \frac{d \lambda}{d t} \\
& +\frac{\lambda(\lambda-1)(\lambda-t)}{t^{2}(t-1)^{2}}\left[\alpha+\beta \frac{t}{\lambda^{2}}+\gamma \frac{t-1}{(\lambda-1)^{2}}+\delta \frac{t(t-1)}{(\lambda-t)^{2}}\right] .
\end{aligned}
$$

Due to its connection with many different disciplines in mathematics and physics, PVI (3.1) has been extensively studied in the past several decades. See [5, 6, 7, 9] and references therein.

One of the fundamental properties for PVI (3.1) is the so-called Painlevé property which says that any solution $\lambda(t)$ of (3.1) has neither movable branch points nor movable essential singularities; in other words, for any $t_{0} \in \mathbb{C} \backslash\{0,1\}$, either $\lambda(t)$ is holomorphic at $t_{0}$ or $\lambda(t)$ has a pole at $t_{0}$. Then it is reasonable to lift PVI (3.1) to the covering space $\mathbb{H}=\{\tau \mid \operatorname{Im} \tau>0\}$ of $\mathbb{C} \backslash\{0,1\}$ by the following transformation:

$$
t=\frac{e_{3}(\tau)-e_{1}(\tau)}{e_{2}(\tau)-e_{1}(\tau)}, \lambda(t)=\frac{\wp(p(\tau) \mid \tau)-e_{1}(\tau)}{e_{2}(\tau)-e_{1}(\tau)} .
$$

Consequently, $p(\tau)$ satisfies the following elliptic form of PVI (cf. [1, 8]):

$$
\frac{d^{2} p(\tau)}{d \tau^{2}}=\frac{-1}{4 \pi^{2}} \sum_{k=0}^{3} \alpha_{k} \wp^{\prime}\left(p(\tau)+\frac{\omega_{k}}{2} \mid \tau\right),
$$

with parameters $\left(\alpha_{0}, \alpha_{1}, \alpha_{2}, \alpha_{3}\right)=\left(\alpha,-\beta, \gamma, \frac{1}{2}-\delta\right)$. The Painlevé property of PVI (3.1) implies that function $\wp(p(\tau) \mid \tau)$ is a single-valued meromorphic function in $\mathbb{H}$.

In this note, we only consider the special case $\alpha_{0}=\frac{9}{8}$ and $\alpha_{k}=\frac{1}{8}$ for $k \geq 1$, i.e.

$$
\frac{d^{2} p(\tau)}{d \tau^{2}}=\frac{-1}{4 \pi^{2}}\left(\frac{9}{8} \wp^{\prime}(p(\tau) \mid \tau)+\sum_{k=1}^{3} \frac{1}{8} \wp^{\prime}\left(p(\tau)+\frac{\omega_{k}}{2} \mid \tau\right)\right)
$$


which is the elliptic form of $\operatorname{PVI}\left(\frac{9}{8}, \frac{-1}{8}, \frac{1}{8}, \frac{3}{8}\right)$.

It is well known (cf. [9, 10, 3]) that any solution of $\operatorname{PVI}\left(\frac{9}{8}, \frac{-1}{8}, \frac{1}{8}, \frac{3}{8}\right)$ can be obtained from that of $\operatorname{PVI}\left(\frac{1}{8}, \frac{-1}{8}, \frac{1}{8}, \frac{3}{8}\right)$ (i.e. the case studied by Hitchin [5]) by the Okamoto transformation [9]. By this way, the following result gives explicit expressions of some solutions of the elliptic from (3.4).

Theorem A. [10, 3] For any $k \in\{0,1,2,3\}$ and $C \in \mathbb{C} \cup\{\infty\}, p_{C}^{(k)}(\tau)$ is a solution of the elliptic form (3.4), where $\wp\left(p_{C}^{(k)}(\tau) \mid \tau\right)$ are given by:

$$
\begin{aligned}
& \wp\left(p_{C}^{(0)}(\tau) \mid \tau\right)= \\
& \frac{2 g_{3}(\tau)(C-\tau)^{3}-4\left(C \eta_{1}(\tau)-\eta_{2}(\tau)\right)^{3}-g_{2}(\tau)\left(C \eta_{1}(\tau)-\eta_{2}(\tau)\right)(C-\tau)^{2}}{(C-\tau)\left[12\left(C \eta_{1}(\tau)-\eta_{2}(\tau)\right)^{2}-g_{2}(\tau)(C-\tau)^{2}\right]},
\end{aligned}
$$

and

$$
\wp\left(p_{C}^{(k)}(\tau) \mid \tau\right)=\frac{\left(\frac{g_{2}(\tau)}{2}-3 e_{k}(\tau)^{2}\right)\left(C \eta_{1}(\tau)-\eta_{2}(\tau)\right)+\frac{g_{2}(\tau)}{4} e_{k}(\tau)(C-\tau)}{3 e_{k}(\tau)\left(C \eta_{1}(\tau)-\eta_{2}(\tau)\right)+\left(\frac{g_{2}(\tau)}{2}-3 e_{k}(\tau)^{2}\right)(C-\tau)}
$$

with $k \in\{1,2,3\}$. Equivalently,

$$
\lambda_{C}^{(k)}(t):=\frac{\wp\left(p_{C}^{(k)}(\tau) \mid \tau\right)-e_{1}(\tau)}{e_{2}(\tau)-e_{1}(\tau)}, t=\frac{e_{3}(\tau)-e_{1}(\tau)}{e_{2}(\tau)-e_{1}(\tau)}
$$

is a Riccati type solution of $\operatorname{PVI}\left(\frac{9}{8}, \frac{-1}{8}, \frac{1}{8}, \frac{3}{8}\right)$.

We recall [10, 2] that the elliptic form (3.4) governs the isomonodromic deformation of GLE (1.7). More precisely,

Theorem B. [2] $p(\tau)$ is a solution of (3.4) if and only if there exists $A(\tau)$ (Consequently, $B(\tau)$ is determined by $(p(\tau), A(\tau))$ via (1.3) with $\left(n_{0}, n_{1}, n_{2}, n_{3}\right)=$ $(1,0,0,0))$ such that GLE (1.7) with $(p, A, B, \tau)=(p(\tau), A(\tau), B(\tau), \tau)$ preserves the monodromy as $\tau$ deforms.

We call such GLE (1.7) with $(p, A, B)=(p(\tau), A(\tau), B(\tau))$ to be the corresponding GLE of the solution $p(\tau)$.

Remark 3.1. The formulas (3.5)-(3.6) were derived in [10, 2] but the monodromy of the corresponding GLE (1.7) was not thoroughly discussed. In [4], it was proved that for $p_{C}^{(k)}(\tau)$, the monodromy of the corresponding GLE (1.7) is not completely reducible (i.e. the form of (1.6)) but without proving that the monodromy data is precisely the same $C$. This assertion, which is strongly suggested by Theorem 1.1, will be proved in following.

Proof of Theorem 1.2. Fix $\tau_{0} \in \mathbb{H}$ and $p_{0} \notin E_{\tau_{0}}[2]$.

Suppose $C \in \mathbb{C} \cup\{\infty\}$ satisfies the cubic equation (1.8) with $\tau=\tau_{0}$ and $p=p_{0}$. Our goal is to prove the existence of $A_{0} \in \mathbb{C}$ such that (1.11) holds for the GLE (1.7) with $(p, A, \tau)=\left(p_{0}, A_{0}, \tau_{0}\right)$. 
Note that $\wp\left(p_{0} \mid \tau_{0}\right)=\wp\left(p_{C}^{(0)}\left(\tau_{0}\right) \mid \tau_{0}\right)$, where $p_{C}^{(0)}(\tau)$ is a solution of the elliptic form (3.4) given in Theorem A, so without loss of generality, we may assume $p_{0}=p_{C}^{(0)}\left(\tau_{0}\right)$ in $E_{\tau_{0}}$. Then by Theorem $\mathrm{B}$ and Remark 3.1 , there is $A(\tau)$ such that the corresponding GLE (1.7) with $(p, A, \tau)=\left(p_{C}^{(0)}(\tau), A(\tau)\right.$, $\tau)$ is monodromy preserving with not completely monodromy representation as long as $p_{C}^{(0)}(\tau) \notin E_{\tau}[2]$. Fix any $\tau \in \mathbb{H}$ such that $p_{C}^{(0)}(\tau) \notin E_{\tau}[2]$. For GLE (1.7) with $(p, A, \tau)=\left(p_{C}^{(0)}(\tau), A(\tau), \tau\right)$, by repeating the proof of Theorem 1.1, there exists a monodromy data $C_{1}=C_{1}(\tau)$ such that either

$$
\begin{aligned}
& \wp\left(p_{C}^{(0)}(\tau) \mid \tau\right)= \\
& \frac{2 g_{3}(\tau)\left(C_{1}-\tau\right)^{3}-4\left(C_{1} \eta_{1}(\tau)-\eta_{2}(\tau)\right)^{3}-g_{2}(\tau)\left(C_{1} \eta_{1}(\tau)-\eta_{2}(\tau)\right)\left(C_{1}-\tau\right)^{2}}{\left(C_{1}-\tau\right)\left[12\left(C_{1} \eta_{1}(\tau)-\eta_{2}(\tau)\right)^{2}-g_{2}(\tau)\left(C_{1}-\tau\right)^{2}\right]}
\end{aligned}
$$

or

$$
\wp\left(p_{C}^{(0)}(\tau) \mid \tau\right)=\frac{\left(\frac{g_{2}(\tau)}{2}-3 e_{k}(\tau)^{2}\right)\left(C_{1} \eta_{1}(\tau)-\eta_{2}(\tau)\right)+\frac{g_{2}(\tau)}{4} e_{k}(\tau)\left(C_{1}-\tau\right)}{3 e_{k}(\tau)\left(C_{1} \eta_{1}(\tau)-\eta_{2}(\tau)\right)+\left(\frac{g_{2}(\tau)}{2}-3 e_{k}(\tau)^{2}\right)\left(C_{1}-\tau\right)}
$$

with some $k \in\{1,2,3\}$ holds for any $\tau$ satisfying $p_{C}^{(0)}(\tau) \notin E_{\tau}[2]$. Since GLE (1.7) with $(p, A, \tau)=\left(p_{C}^{(0)}(\tau), A(\tau), \tau\right)$ is monodromy preserving, so the monodromy data $C_{1}$ is a constant dependent of $\tau$. By comparing the RHS of (3.5) and (3.7)- (3.8) which hold for any $\tau$ satisfying $p_{C}^{(0)}(\tau) \notin E_{\tau}[2]$ (i.e. any $\tau \in \mathbb{H}$ except a discrete set), we easily conclude that (3.7) and $C_{1}=C$ hold. Then by letting $A_{0}=A\left(\tau_{0}\right)$ and using $p_{0}=p_{C}^{(0)}\left(\tau_{0}\right)$, we see that $C$ is precisely the monodromy data of GLE (1.7) with $(p, A, \tau)=\left(p_{0}, A_{0}, \tau_{0}\right)$ and $\left(\varepsilon_{1}, \varepsilon_{2}\right)=(1,1)$. This proves $(1)$.

The assertion (2) can be proved similarly.

The above proof highlights the effectiveness of Painlevé VI equation to solve such kind of Riemman-Hilbert problems. We will apply this idea to consider the general case $n_{k} \in \mathbb{Z}$ for all $k$ in a future work.

\section{REFERENCES}

[1] M. V. Babich and L. A. Bordag; The elliptic form of the sixth Painleve equation. Preprint NT Z25/1997, Leipzig (1997).

[2] Z. Chen, T.J. Kuo and C.S. Lin; Hamiltonian system for the elliptic form of Painlevé VI equation. J. Math. Pures Appl. 106 (2016), 546-581.

[3] Z. Chen, T.J. Kuo and C.S. Lin; Simple zero property of some holomorphic functions on the moduli space of tori. preprint, 2016.

[4] Z. Chen, T.J. Kuo and C.S. Lin; Painlevé VI equation, modular forms and application. preprint, 2016.

[5] N. J. Hitchin; Twistor spaces, Einstein metrics and isomonodromic deformations. J. Differ. Geom. 42 (1995), no.1, 30-112. 
[6] K. Iwasaki, H. Kimura, S. Shimomura and M. Yoshida; From Gauss to Painlevé: A Modern Theory of Special Functions. Springer vol. E16, 1991.

[7] O. Lisovyy and Y. Tykhyy; Algebraic solutions of the sixth Painlevé equation. J. Geom. Phys. 85 (2014), 124-163.

[8] Y. Manin; Sixth Painlevé quation, universal elliptic curve, and mirror of $\mathbb{P}^{2}$. Amer. Math. Soc. Transl. (2), 186 (1998), 131-151.

[9] K. Okamoto; Studies on the Painlevé equations. I. Sixth Painlevé equation $P_{V I}$. Ann. Mat. Pura Appl. 146 (1986), 337-381.

[10] K. Takemura; The Hermite-Krichever Ansatz for Fuchsian equations with applications to the sixth Painlevé equation and to finite gap potentials. Math. Z. 263 (2009), 149-194.

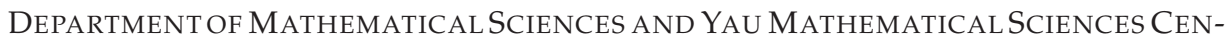
TER, BEIJING, 100084, CHINA

E-mail address: $\mathrm{z}$ jchen@math.tsinghua. edu.cn

TAida Institute for MAthematical SCiEnCEs (TIMS), NATiONAl TAiWAN UniVERSITY, TAIPEI 10617, TAIWAN

E-mail address: tjkuo1215@gmail.com

TAida Institute for MAthematical Sciences (TIMS), CEnter For Advanced Study in Theoretical Sciences (CASTS), National TaiWAN University, Taipei 10617, TAIWAN

E-mail address: cslinemath. ntu.edu.tw

School of Mathematics, University of LeEds, LeEds LS2 9JT, UNited KingDOM, Department of MATHEMATICs, FACUlty of Science AND ENGINEERING, CHuO UNIVERSITY, 1-13-27 KASUGA, BUNKYO-KU TOKYO 112-8551, JAPAN

E-mail address: takemura@math.chuo-u.ac.jp 